\title{
Uma leitura da violência doméstica contra a mulher em tempos de pandemia da
}

\section{Covid 19}

\author{
A reading of domestic violence against women in times of Covid 19 pandemic \\ Una lectura de la violencia doméstica contra las mujeres en tiempos de la pandemia del Covid 19
}

Recebido: 07/02/2022 | Revisado: 14/02/2022 | Aceito: 23/02/2022 | Publicado: 04/03/2022

Ana Virgínia Nunes Soares ORCID: https://orcid.org/0000-0001-5339-8655 Centro Universitário Maurício de Nassau, Brasil E-mail: avnsoares@hotmail.com

Laís de Meneses Carvalho Arilo ORCID: https://orcid.org/0000-0002-1625-5601 Centro Universitário Santo Agostinho, Brasil E-mail: lais.arilo@hotmail.com

Lizandra da Silva Sousa ORCID: https://orcid.org/0000-0001-8363-5914 Centro Universitário Maurício de Nassau, Brasil E-mail: sousalizandra12@gmail.com

Sabrina Amorim Paulo ORCID: https://orcid.org/0000-0002-9339-0612 Centro Universitário Maurício de Nassau, Brasil E-mail: samorimpaulo@gmail.com

Aylana Thaiane de Sousa Vale ORCID: https://orcid.org/0000-0002-7709-0812 Centro Universitário Maurício de Nassau, Brasil E-mail: aylanatsv@hotmail.com

\begin{abstract}
Resumo
O objetivo desta pesquisa é analisar os impactos da pandemia da Covid 19 na violência doméstica praticada contra a mulher. Optou-se pela pesquisa baseada no levantamento de dados oriundos do Fórum Brasileiro de Segurança Pública (FBSP) e pela coleta de informações em jornais, revistas e vídeos veiculados em meio eletrônico. A violência contra a mulher é um problema multifatorial e sistêmico, envolvendo alguns determinantes como: questões culturais e de gênero, vulnerabilidade social e raça. No Brasil, nove em cada dez casos de violência contra a mulher ocorrem no ambiente doméstico. O recorte temporal utilizado foi o período de março e abril de 2020, comparados com o mesmo período do ano anterior. Os dados coletados apontam que existe uma significativa relação entre o aumento da violência doméstica contra a mulher e o distanciamento social, como medida sanitária para prevenção da Covid 19. Foi possível inferir um aumento expressivo do número de feminicídios e um incremento nas denúncias por meio das redes sociais e por canais de denúncia do disque 180 e 190. Por outro lado, os registros de denúncia que dependem da presença física da mulher apresentaram queda durante o período pesquisado. É possível concluir que houve um aumento expressivo de casos de violência doméstica contra a mulher no período pesquisado e a importância de que sejam intensificadas intervenções para fortalecer as políticas públicas de proteção e enfrentamento específico dessa temática.
\end{abstract}

Palavras-chave: Violência doméstica contra a mulher; Pandemia; Covid 19.

\begin{abstract}
The objective of this research is to analyze the impacts of the Covid 19 pandemic on domestic violence against women. We opted for the research based on the collection of data from the Brazilian Forum of Public Security (FBSP) and the collection of information in newspapers, magazines and videos broadcast in electronic media. Violence against women is a multifactorial and systemic problem, involving some determinants such as: cultural and gender issues, social vulnerability and race. In Brazil, nine out of ten cases of violence against women occur in the domestic environment. The time frame used was the period of March and April 2020, compared to the same period of the previous year. The data collected indicate that there is a significant relationship between the increase in domestic violence against women and social distancing, as a health measure to prevent Covid 19. It was possible to infer a significant increase in the number of femicides and an increase in complaints through the social networks and through the hotline 180 and 190 reporting channels. On the other hand, the number of complaints that depend on the physical presence of women dropped during the period surveyed. It is possible to conclude that there was a significant increase
\end{abstract}


in cases of domestic violence against women in the researched period and the importance of intensifying interventions to strengthen public policies for the protection and specific confrontation of this issue.

Keywords: Domestic violence against women; Pandemic; Covid 19.

\section{Resumen}

El objetivo de esta investigación es analizar los impactos de la pandemia del Covid 19 en la violencia doméstica contra las mujeres. Optamos por la investigación basada en la recopilación de datos del Foro Brasileño de Seguridad Pública (FBSP) y la recopilación de informaciones en periódicos, revistas y videos difundidos en medios electrónicos. La violencia contra las mujeres es un problema multifactorial y sistémico, que involucra algunos determinantes como: cuestiones culturales y de género, vulnerabilidad social y raza. En Brasil, nueve de cada diez casos de violencia contra la mujer ocurren en el ámbito doméstico. El marco temporal utilizado fue el período de marzo y abril de 2020, en comparación con el mismo período del año anterior. Los datos recabados indican que existe una relación significativa entre el aumento de la violencia doméstica contra la mujer y el distanciamiento social, como medida sanitaria para prevenir el Covid 19. Se pudo inferir un aumento significativo en el número de feminicidios y un aumento en las denuncias a través de las redes sociales ya través de los canales de denuncia 180 y 190 de la línea directa. Por otro lado, el número de denuncias que dependen de la presencia física de la mujer disminuyó durante el período encuestado. Es posible concluir que hubo un aumento significativo de los casos de violencia doméstica contra la mujer en el período investigado y la importancia de intensificar las intervenciones para fortalecer las políticas públicas de protección y enfrentamiento específico de esta problemática.

Palabras clave: Violencia doméstica contra la mujer; Pandemia; Covid 19.

\section{Introdução}

A violência contra a mulher constitui-se em um problema de saúde pública e de grave violação aos direitos humanos, em especial ao direito à vida sem violência. A prática da violência no ambiente doméstico por parceiro íntimo traz uma série de consequências negativas para a mulher, provocando um grave adoecimento físico, mental e social, com sequelas expressivas como: medo, angústia, ansiedade, depressão, distúrbios do sono, síndrome do pânico, estresse pós- traumático, problemas gastrintestinais, dores no corpo, agravos de natureza ginecológica, tabagismo, consumo de álcool, distúrbios alimentares, dificuldades para realizar tarefas cotidianas, baixa autoestima, dificuldades relacionadas à sexualidade, até consequências mais graves como a ideação suicida (Word Health Organization, 2017).

Segundo a Organização Mundial da Saúde (2020) essas mulheres apresentam ainda maior frequência no uso de serviços do setor de saúde e maiores agravamentos, em especial, os de saúde mental. Além de estarem sujeitas a discriminações e julgamentos e perda de seus postos de trabalho. Ademais, as consequências dessa violência repercutem em sintomas físicos e psíquicos, de logo prazo, podendo até mesmo se tornarem crônicos (Campos et., al. 2021).

Segundo Pasinato (2018), a violência contra a mulher foi naturalizada e tolerada ao longo dos tempos em nosso país. A reprodução dos estereótipos de gênero e a manutenção de paradigmas e valores de uma sociedade sexista e permeada pelo machismo estrutural proporcionaram uma construção histórica de violação dos direitos da mulher. O Brasil possui dois diplomas legais específicos para o enfrentamento e erradicação da violência doméstica praticada contra a mulher. O primeiro deles, a Lei Maria da Penha, é uma legislação com características não apenas punitivas, mas também é uma ação afirmativa em favor das mulheres vítimas de violência doméstica. É uma lei de prevenção que demanda um conjunto de ações e políticas nas áreas de saúde, segurança, educação, assistência social, apoio psicológico e jurídico voltadas para a mulher vítima de violência (Lei n. 11.340, 2006).

A Lei Maria da Penha enumera cinco formas de violência contra a mulher, sem excluir outras possibilidades. Contempla a violência psicológica, moral, patrimonial, física e sexual e traz um sistema integrado de proteção para a mulher vítima de violência, com previsão de mecanismos como a concessão de medidas protetivas de urgência, além de políticas públicas voltadas à ressocialização, reeducação e grupos de reflexão para o homem autor de violência. A evolução de todas essas formas de violência doméstica contra a mulher atinge sua forma mais grave e letal no feminicídio, fruto de uma violência que decorre de uma construção desigual de gênero (Pasinato, 2008). 
A Lei n. 13.104/15 regulamenta o feminicídio no Brasil. É o segundo diploma legal específico para enfrentamento da violência doméstica. A violência praticada contra a mulher insere o país na quinta posição na escala de países que mais matam mulheres por questões de gênero, pelo só fato de serem mulheres (Organização das Nações Unidas, 2016).

A violência doméstica contra a mulher é oriunda de um processo que se intensifica desde o ano de 2017. Pesquisa publicada no ano de 2019 pelo Fórum Brasileiro de Segurança Pública (FBSP) em parceria com o Instituto de Pesquisa Econômica Aplicada (IPEA), analisando dados de 2017 e 2018, indicou que do total de homicídios contra mulheres, 28,5\% ocorrem dentro da residência, o que evidencia que a casa da mulher vítima de violência doméstica num contexto de não pandemia já demonstra ser um lugar não seguro (Atlas da Violência, 2019).

Por ser um problema de vasto alcance no Brasil e no mundo, a violência contra a mulher, dentre uma plêiade de outras questões, tem sido palco de discussões e possíveis estratégias de enfrentamento, ante o árduo e desfavorável cenário que assolou o planeta nos últimos meses: a chegada da pandemia do novo coronavírus. A Covid 19 tem provocado uma crise em diversos setores do país com o consequente agravamento de problemas sociais e de saúde pública já existentes, tendo a violência doméstica contra a mulher como um dos mais visíveis para o momento.

Nesse contexto, buscou-se analisar como o distanciamento social de mulheres vítimas de violência doméstica durante o período da pandemia da Covid 19, contribui para o aumento dos casos de violência e como colocá-lo em prática sem comprometer a integridade física, moral, psicológica e a própria vida das mulheres que se encontram em relacionamentos abusivos. Nesse sentido, o ambiente doméstico ao invés de ter sido um ambiente de proteção, tem se torna um ambiente cercado de violência (Nascimento et al., 2021).

O objetivo desta pesquisa é fazer um levantamento de dados sobre os impactos da pandemia da Covid 19 e do distanciamento social, enquanto medida sanitária adotada para prevenção da doença, na vida de mulheres em situação de violência doméstica, discutindo e avaliando se esse novo cenário pode ou não interferir no aumento da violência doméstica praticada contra a mulher.

\section{Metodologia}

Para a construção da investigação proposta no presente artigo, optou-se pela pesquisa baseada no levantamento de dados sobre a violência doméstica contra a mulher oriundos do estudo realizado pelo Fórum Brasileiro de Segurança Pública (FBSP), mediante solicitação do Banco Mundial, em doze estados da federação, no período referente aos meses de março e abril de 2019, comparativamente ao mesmo período de 2020, e pela pesquisa de dados relativos ao tema em jornais, revistas e vídeos publicados em meio eletrônico no período de março a abril de 2020, momento em que se iniciou a medida sanitária do distanciamento social na maioria dos estados brasileiros e no mundo.

Segundo Gil (2008), a pesquisa de levantamento de dados é a interrogação direta das pessoas cujo comportamento se deseja conhecer. Procede-se à solicitação de informações a um grupo significativo de pessoas acerca do problema estudado para, em seguida, mediante análise quantitativa, obterem-se as conclusões correspondentes aos dados coletados.

Para a confecção deste artigo, utilizou-se como critério de inclusão os dados colhidos pelo FBSP e as informações constantes de jornais, revistas e vídeos veiculados em meios eletrônicos que refletem a violência contra a mulher adulta praticada no ambiente doméstico por parceiro íntimo em relações heteroafetivas, ocorrida no período de março e abril de 2020 , comparadas com o mesmo lapso temporal do ano anterior. Os critérios de exclusão referem-se a outras modalidades de violência praticadas contra a mulher fora do ambiente doméstico, que não decorram de relações íntimas de afeto e estejam fora do período pesquisado. 


\section{Resultados e Discussão}

O cenário mundial sinaliza para um aumento da violência doméstica contra a mulher em vários países. O secretário geral da ONU em pronunciamento no mês de abril de 2020, fez um alerta para os impactos específicos da Covid 19 nas mulheres e para o aumento da violência contra a mulher em meio à pandemia. Também fez um apelo para todos os governos no sentido de que façam das medidas de prevenção em caso de violência contra a mulher uma parte essencial dos seus planos nacionais de resposta à Covid19 (Agência ONU Brasil, 2020)

De acordo com dados do Fórum Brasileiro de Segurança Pública (2020), na Itália, os primeiros dados de registros policiais indicaram redução de $43 \%$ nas denúncias/ocorrências de violência doméstica. Na China, primeiro país a detectar o vírus em sua população, as denúncias de violência doméstica triplicaram no período do confinamento quando comparados com dados do mesmo período do ano anterior. Na França e Espanha, foram registrados aumentos dos casos e subnotificação das denúncias.

Diante destes números que revelam um grave aumento de casos de abusos e agressões no ambiente doméstico contra a mulher, os governos estrangeiros passaram a adotar um conjunto de medidas diversificadas na tentativa de conter o avanço da violência doméstica. O governo francês instalou um sistema de senhas emergenciais nas farmácias para o socorro imediato à mulher vítima de violência doméstica por parte dos serviços e ações de proteção e fez um acordo com a rede hoteleira para alugar quartos de hotéis para abrigar essas mulheres.

A exemplo da França, a Itália também decidiu converter quartos de hotéis em abrigos para que as mulheres em situação de violência cumpram as medidas de distanciamento social de forma segura e longe de seus agressores. Nesse país, a polícia adotou aplicativos para denunciar casos de violência doméstica, com a possibilidade de envio de mensagens e fotos, sem que o parceiro da vítima tenha conhecimento. Já a Espanha, onde também foi registrado um aumento dos números de casos e uma subnotificação das denúncias, adotou ferramentas de denúncias por mensagem de geolocalização, via WhatsApp, e o governo espanhol declarou como essenciais os serviços de atendimento às mulheres vítimas de violência, além de utilizar quartos de hotéis como abrigo (Fórum Brasileiro De Segurança Pública - FBSP, 2020).

No cenário brasileiro, a análise da violência doméstica contra a mulher durante a pandemia da Covid 19, de acordo com os dados da pesquisa realizada pelo Fórum Brasileiro de Segurança Pública -FBSP (2020), revela que os boletins de ocorrência realizados por meio da presença física da mulher nas Delegacias Especializadas de Atendimento à Mulher (DEAM) apresentaram queda nos primeiros dias de isolamento. Isso se verifica pelo fato da maior dificuldade da mulher de se deslocar à uma delegacia em razão do medo de sair de casa para registrar a ocorrência, tanto pela vigilância constante e excessiva do agressor como pela percepção que ela possui a respeito da possibilidade de contaminação pelo vírus.

Foi possível verificar uma redução média de $25,5 \%$ dos registros de lesão corporal dolosa em decorrência de violência doméstica no período de março e abril de 2020, em relação ao mesmo período de 2019. A maior redução se deu no Maranhão, com 97,3\% de redução entre março e abril desse ano. No Rio de Janeiro, a redução no número de registros de agressão de violência doméstica que resultaram no crime de lesão corporal de natureza dolosa foi de 48,5\% e no Pará de 47,8\%. O Estado do Rio Grande do Norte, único que apresentou crescimento no número de registros em março desse ano verificou uma queda de 57,7\% das denúncias presenciais nas DEAMS em abril, com as medidas de distanciamento social em vigor.

Quanto à taxa de feminicídios, a análise dos dados publicados pelo FBSP revela um crescimento nos 12 estados pesquisados. O aumento verificado foi de $22,2 \%$ no país, passando de 117 vítimas em março/abril de 2019 para 143 vítimas em março/abril de 2020. No Acre, o crescimento chegou a 300\%, passando de 1 para 4 vítimas este ano; no Maranhão, o crescimento foi de 166,7\%, de 6 para 16 vítimas. No Estado do Mato Grosso, o crescimento foi de $150 \%$, passando de 6 para 
15 vítimas. Apenas três unidades da federação registraram redução no número de feminicídios no período: Minas Gerais (22,7\%), Espírito Santo (-50\%), e Rio de Janeiro (-55,6\%) (Agência Brasil,2020).

As denúncias no disque 180, número da Central de Atendimento à Mulher em Situação de Violência Doméstica, disponibilizado pelo Ministério da Mulher, da Família e dos Direitos Humanos, do Governo Federal, também registraram um aumento no número de ligações reportando casos de violência doméstica. De acordo com os dados registrados na pesquisa do FBSP, nos meses de março e abril nos últimos três anos, foi possível verificar um crescimento contínuo de denúncias por este canal. Apenas em abril deste ano, quando comparado com o número de denúncias de abril de 2019, o crescimento foi de 37,6\%, revelando o incremento da violência contra a mulher no período (Fórum Brasileiro De Segurança Pública - FBSP, 2020).

Quanto às denúncias registradas pelo disque 190, número de acesso à Polícia Militar em todo o Brasil, dos doze estados analisados na pesquisa do FBSP sobre a violência doméstica contra a mulher no período da pandemia da Covid-19, apenas três estados forneceram dados sobre os registros de violência realizados através deste canal: Acre, São Paulo e Rio de Janeiro. Em São Paulo, o crescimento entre março de 2019 e março de 2020 foi de 44\%. O estado do Acre apresentou crescimento médio de 19,6\% nos quatro primeiros meses deste ano quando comparado com o mesmo período do ano passado, acentuando-se no mês de abril, com crescimento de 33\%. No Rio de Janeiro, no mês de abril, período em que já vigoravam medidas mais restritivas de isolamento social no Estado, o crescimento foi de 5,1\% nas denúncias realizadas pelo número da Polícia Militar.

Dados do Fórum Brasileiro de Segurança Pública, oriundos de monitoramento no Twitter, entre os meses de fevereiro e abril desse ano, apontam para um aumento $431 \%$ de postagens por vizinhos relatando brigas de casal com indícios de violência doméstica, em relação a períodos em que não vivemos o distanciamento social. Do total de relatos nas postagens, restou evidenciado que o dia da semana mais violento é a sexta feira, com aproximadamente $25 \%$ das postagens, concentrando-se na madrugada, entre 20h e 03 horas da manhã (Fórum Brasileiro De Segurança Pública - FBSP, 2020).

Em alguns estados da federação foi implantado o Boletim de Ocorrência Eletrônico, a exemplo do Estado do Piauí. Nesse Estado, a Secretaria de Segurança Pública também desenvolveu um aplicativo de celular para denúncias de violência contra a mulher, chamado "Salve Maria". O aplicativo permite que qualquer pessoa mande denúncias com vídeo, foto, áudio e texto com informações sobre a vítima e o agressor. Cresceu em 32\% o número de pedidos de socorro feitos através do botão do pânico do Salve Maria, durante o mês de março deste ano. Segundo a Secretaria de Segurança Pública, em março de 2019 foram feitos 53 chamados de socorro através do aplicativo. Em março de 2020, o número subiu para 70 casos - pelo menos dois casos de violência por dia (Globo, 2020).

Ainda, segundo a Secretaria de Segurança Pública deste Estado, em março de 2020 foram registrados 593 crimes pelas delegacias especializadas no atendimento às mulheres. O número é $29 \%$ menor do que o de março de 2019, quando foram registrados 841 crimes. Essa redução é vista como um reflexo do isolamento, que teria causado uma queda nas denúncias de violência pela forma presencial (G1, Globo, 2020). Outra possibilidade no enfrentamento da violência doméstica contra a mulher no período da pandemia é o encaminhamento para a Casa da Mulher Brasileira, equipamento de apoio, proteção e acolhimento à mulher vítima de violência. A Casa da Mulher Brasileira possui unidades em funcionamento em seis unidades da federação: Campo Grande (MS), São Luís (MA), Boa Vista (RR), Fortaleza (CE), Curitiba (PR) e São Paulo (SP), e previsão de implantação de mais 25 unidades em 2020. Possui competência nacional, atendendo mulheres por meio de uma rede multidisciplinar de profissionais e órgãos que compõem o sistema de justiça (Defensoria Pública do Estado do Piaú́, 2020).

No sentido de valorizar ainda mais os canais de denúncia no período da pandemia, o Estado de São Paulo, através do Ministério Público, criou uma rede de estratégias e ações de combate à violência doméstica, por meio da implantação da plataforma digital "Justiceiras Oficial". As vítimas de violência são direcionadas, via WhatsApp, para uma equipe de 
atendimento multidisciplinar e, até o momento, de acordo com dados fornecidos pela plataforma, 650 vítimas já foram atendidas (Globoplay, 2020).

Os resultados apresentados no decorrer deste artigo sugerem que existe uma significativa relação entre o aumento da violência doméstica e o distanciamento social, como medida sanitária para prevenir a Covid 19, nas capitais brasileiras pesquisadas. A análise da nota técnica do Fórum Brasileiro de Segurança Pública, publicada no final do mês de maio de 2020, permite inferir um aumento expressivo no número de feminicídios cometidos, além do aumento no número de denúncias de agressão pelos canais do disque 180 e 190. O incremento de denúncias por meio das redes sociais também pode ser verificado. Por outro lado, os registros de denúncia que dependem da presença física da mulher nas DEAM apresentaram queda, dado previsível em razão da maior dificuldade de deslocamento da vítima em função da vigilância constante e exacerbada do agressor, oriundas do convívio contínuo decorrente do confinamento social.

\section{Considerações Finais}

A violência doméstica contra a mulher é resultado de todo um processo histórico e cultural de exclusão, sulbartenidade, objetificação e imposição de papéis de gênero construídos socialmente ao longo de décadas. Uma realidade que não é específica do Brasil, mas que a cada dia assume proporções maiores fazendo com que já se viva uma pandemia que exclui a mulher da condição de sujeito de direitos e viola seu direito humano básico a uma vida sem violência.

O momento atípico provocado pela pandemia da Covid 19 requer da sociedade e do Estado um olhar mais diligente para a violência doméstica praticada contra a mulher. Orientar o que fazer é o mais importante no momento e direcionar a mulher vítima de violência para os centros e instituições que fazem parte da rede de apoio. A informação e a conscientização a respeito dessa grave violação de direitos constituem-se numa das vias possíveis para propiciar uma cultura de respeito e igualdade de gênero.

O fato de a mulher vítima de violência doméstica ter a informação de que os serviços de proteção e acolhimento e os canais de denúncia continuam funcionando no período do isolamento social, inclusive, de forma digital para facilitar o acesso, certamente constituirá para elas um reforço protetivo. A partir deste estudo, verificou-se a importância de que sejam intensificadas, nesse momento de confinamento social imposto pela pandemia da Covid 19, as intervenções para minimizar os casos de violência doméstica contra a mulher, fortalecendo e dando continuidade às políticas públicas de proteção e enfrentamento específico dessa temática já existentes, bem assim, a presença do sistema de justiça para dar efetividade ao cumprimento das leis de proteção às vítimas de violência doméstica.

Como limitações neste estudo, destaca-se o recorte temporal pesquisado referente ao primeiro trimestre de 2020, comparativamente ao mesmo período do ano anterior, o que possibilitou apenas a coleta de dados iniciais referentes ao tema. Sugere-se a realização de novos estudos no sentido de avaliar, com maior aprofundamento, o fenômeno da violência doméstica no contexto da pandemia da Covid 19 e suas implicações na saúde física e mental da mulher.

\section{Referências}

Alves, V. (2020). Denúncias de violência doméstica crescem 32\% durante isolamento em Teresina. https://globoplay.globo.com/v/8518012/

Bernardino, E. (2020). A Violência Contra a Mulher em Tempos de Pandemia. 333333 https://www.youtube.com/watch?v=AQLztaUJY1E

Bond, L. (2020). Casos de feminicídio crescem 22\% em 12 estados durante pandemia. Agência Brasil. https://agenciabrasil.ebc.com.br/direitoshumanos/noticia/2020-06/casos-de-feminicidio-crescem-22-em-12-estados-durante-pandemia

Campos, B., Tchalekian, B., \& Paiva, V. (2020). Violência contra a mulher: vulnerabilidade programática em tempos de SARS-COV-2/COVID-19 em São Paulo. Psicologia \& Sociedade, 32. https://doi.org/10.1590/1807-0310/2020v32240336 
Defensoria Pública do Estado do Piauí. (2020). Núcleo de Defesa da Mulher orienta sobre registro de Boletim Eletrônico para mulheres vítimas de violência doméstica. http://www.defensoria.pi.def.br/nucleo-de-defesa-da-mulher-orienta-sobre-registro-de-boletim-eletronico-para-mulheres-vitimas-de-violenciadomestica/

Nascimento, E. F. do., Monte, L. M. I. do, Silva, R. A. dá, Santos, F. K. L. dos, Pessoa, B. G. F., Sousa Junior, P. de T. X.., Araújo, N. J. C., \& Silva, L. D. (2021). With sugar and without affection: Violence against women in the context of Covid-19. Research, Society and Development, 10(5), e27410514696. https://doi.org/10.33448/rsd-v10i5.14696

Fórum Brasileiro De Segurança Pública (FBSP). (2020). Violência Doméstica durante a Pandemia de Covid-19 - ED. 2. https://forumseguranca.org.br/wpcontent/uploads/2020/06/violencia-domestica-covid-19-ed02-v5.pdf

Gil, A. C. (2008). Como elaborar projetos de pesquisa (4a. ed). Atlas.

Governo Federal. (2018). Serviços disponíveis na Casa da Mulher Brasileira. https://www.gov.br/mdh/pt-br/navegue-por-temas/politicas-paramulheres/arquivo/assuntos/violencia/programa-mulher-viver-sem-violencia/servicos-disponiveis-na-casa-da-mulher-brasileira

Governo Federal. (2019). Casa Da Mulher Brasileira É Estratégia Para Reduzir A Violência Contra A Mulher. Recuperado De: Https://Www.Gov.Br/PtBr/Noticias/Assistencia-Social/2019/11/Casa-Da-Mulher-Brasileira-E-Estrategia-Para-Reduzir-A-Violencia-Contra-A-Mulher

Lei $n^{o}$. 11.340, de 7 de agosto de 2006 (Lei Maria da Penha). Cria mecanismos para coibir a violência doméstica e familiar contra a mulher, nos termos do $§ 8^{\circ}$ do art. 226 da Constituição Federal, da Convenção sobre a Eliminação de Todas as Formas de Discriminação contra as Mulheres e da Convenção Interamericana para Prevenir, Punir e Erradicar a Violência contra a Mulher; dispõe sobre a criação dos Juizados de Violência Doméstica e Familiar contra a Mulher; altera o Código de Processo Penal, o Código Penal e a Lei de Execução Penal; e dá outras providências. http://www.planalto.gov.br/ccivil_03/_ato2004-2006/2006/lei/111340.htm

Lei 13.104/05, de 9 de março de 2015. Altera o art. 121 do Decreto-Lei no 2.848, de 7 de dezembro de 1940 - Código Penal, para prever o feminicídio como circunstância qualificadora do crime de homicídio, e o art. $1^{\circ}$ da Lei n ${ }^{\circ} 8.072$, de 25 de julho de 1990, para incluir o feminicídio no rol dos crimes hediondos. http://www.planalto.gov.br/ccivil_03/_ato2015-2018/2015/lei/113104.htm

Modelli, L. (2020). Violência física e sexual contra mulheres aumenta durante isolamento social provocado por coronavírus. G1. https://g1.globo.com/bemestar/coronavirus/noticia/2020/04/19/violencia-fisica-e-sexual-contra-mulheres-aumenta-durante-isolamento-social-provocado-pelocoronavirus.ghtml

Nascimento, A. (2020). Denúncias de violência doméstica pelo Salve Maria crescem 32\% durante isolamento social no Piauí. G1 Piauí. https://g1.globo.com/pi/piaui/noticia/2020/04/29/denuncias-de-violencia-domestica-no-pi-crescem-32percent-durante-isolamento-social-pelo-salvemaria.ghtml

Organização Mundial de Saúde (OMS). (2017). Violence against women. https://www.who.int/en/news-room/fact-sheets/detail/violence-against-women

Organização das Nações Unidas Brasil (ONU Brasil). (2016). ONU: Taxa de feminicídios no Brasil é quinta maior do mundo. Diretrizes nacionais buscam solução. https://nacoesunidas.org/onu-feminicidio-brasil-quinto-maior-mundo-diretrizes-nacionais-buscam-solucao/

Organização das Nações Unidas Brasil (Onu Brasil). (2020). Chefe da Onu Alerta Para Aumento da Violência Doméstica em Meio À Pandemia Do Coronavírus. Https://Nacoesunidas.Org/Chefe-Da-Onu-Alerta-Para-Aumento-Da-Violencia-Domestica-Em-Meio-A-Pandemia-Do-Coronavirus/

Organização das Nações Unidas Brasil (ONU Brasil). (2020). Relatora da ONU: Estados devem combater violência doméstica na quarentena por COVID-19. Recuperado de: https://nacoesuni das.org/relatora-da-onu-estados-devem-combater-violencia-domestica-na-quarentena-por-covid-19/

Organização Pan-Americana de Saúde (OPAS). (2020). COVID-19 e a violência contra a mulher: O que o setor/sistema de saúde podefazer. https://iris.paho.org/bitstream/handle/10665.2/52016/OPASBRACOVID1920042_por.pdf?ua=1.

Pasinato, W. (2018). “As mulheres são vítimas de violência porque são mulheres”. Revista Galileu. https://revistagalileu.globo.com/Sociedade/noticia/2018/02/violencia-contra-mulher-wania-pasinato.html 